\title{
Development of Carrier Based in Vitro Produced Arbuscular Mycorrhizal (AM) Fungal Inocula for Organic Agriculture
}

\author{
Rodrigues Kim Maria* and Rodrigues Bernard Felinov \\ Department of Botany, Goa University, Taleigao Plateau, Goa 403206 India. \\ Email: botany.kim@unigoa.ac.in
}

\begin{abstract}
Studies on the advantageous effects of arbuscular mycorrhizal (AM) fungi are providing new possible ways to exploit them as biofertilizers in sustainable agriculture. Many studies have described the potential of root organ culture (ROC) system for production of AM fungal inocula. However there is a need for development of a suitable carrier formulation to support in vitro produced AM fungal inocula when mixed with substrate, so as to enable the delivery of inocula in the rhizosphere. The aim of this study was to assess the performance of the organic carrier formulation consisting of vermiculite as the main component along with cattle manure, wood powder and wood ash in different proportions; and its ability to retain inoculum potential of the in vitro produced AM fungal propagules of Rhizoglomus intraradices and Funneliformis mosseae. Treatment 5 comprising of carrier formulation (vermiculite: cow dung powder: wood powder: wood ash) in the ratio of 20:8:2:1 was observed to be as the best carrier treatment for both the in vitro produced AM species. The in vitro produced propagules of both AM species were viable and effectively colonized the roots of Eleusine coracana Gaertn. The method established shows the efficiency of the carrier formulation in sustaining the inoculum potential of in vitro produced AM propagules for mass multiplication and possibility in application.
\end{abstract}

Keywords: In vitro, AM fungal propagules, carrier formulation, inoculum.

\section{Introduction}

Arbuscular mycorrhizal (AM) fungi are ubiquitous soil fungi forming mutualistic symbiosis with plant roots. AM fungal extra-radical hyphal network spreading extensively in the soil and acting as an extension to the host's root system in nutrient depletion zones has significant effects on overall host plant growth and development. Efficient exchange of nutrients is mediated via specialized structures within the root cortical cells (arbuscules). The basis of this symbiosis is the ability of AM fungi to form fine extra-radical hyphae in order to increase root-soil contact area as well as secrete enzymes/organic acids for improved nutrient acquisition [1]. In addition to improved uptake of soil minerals, other benefits ascribed to the host plant are improved water relations and disease resistance [2]. The beneficial effects of AM fungi on plant growth and nutrition have led to an increased use of AM fungal inoculum as biofertilizer [3]. Large scale AM fungal inoculum production is precluded due to their obligate biotrophic nature i.e. they must grow in symbiosis with living host plant roots in order to complete their life cycle and to produce infective propagules. AM fungal inoculum is presently produced in a variety of ways utilizing in vitro, greenhouse, or field-based methods [4-6]. The in vitro method comprises of monoxenic culture of sterilized AM fungal spores with Ri T-DNA transformed carrot roots [7]. The root organ culture (in vitro) system is preferred over the classical (pot/trap culture) method, permitting production of pure, viable, contamination free propagules in a smaller space.

AM fungal inoculum is commercially available in a variety of forms ranging from high concentrations of AM fungal propagules in carrier materials to potting media containing inoculum at low concentrations [8]. Biofertilizers are usually prepared as carrier-based inoculants containing effective microorganisms [9]. A carrier is a delivery vehicle which is used to transfer live microorganism from an agar slant to the rhizosphere [10]. A suitable biofertilizer carrier should comprise of certain characteristic features viz., it should be in powder or granular form, should support the growth and survival of the microorganism, should be able to release the functional microorganism easily into the soil, should have high moisture absorption and retention capacity, should have good aeration characteristics and $\mathrm{pH}$ 
buffering capacity [11]. Besides it should be non-toxic and environmentally friendly, should be easily sterilized and handled in the field, have good long term storage qualities, and should be inexpensive [12, 13]. Considering the above mentioned features it is apparent that not a solitary universal carrier is available which fulfills all the desirable characteristics, but good quality ones should have as many as possible.

AM fungal inoculum comprises of spores, colonized root fragments and mycelium/hyphae. Isolated AM fungal spores and hyphae can then be mixed with the carrier material. The carrier materials can be organic, inorganic or synthetic. Commonly used carriers include soils like peat, coal, pumice or clay, sand, and lignite; inert materials like perlite, vermiculite, soilrite, alginate beads, polyacrylamide gels and bentonite [14-19]. Organic wastes from animal production and agriculture, and byproducts of agricultural and food processing industries such as charcoal, composts, farmyard manure, cellulose, soybean meal, soybean and peanut oil, wheat bran, press mud, corn cobs also meet the requirements of a biofertilizer carrier and thus could be good carrier materials [20, 21]. It is also possible to find carrier combinations comprising of a mixture of soil and compost; soil, peat, bark, and husks among others [18]. Peat is the most commonly used carrier material. However, it is a limited natural resource which is not readily available worldwide and its use has a detrimental effect on the environment from which it is extracted. This highlights the need for development of new carrier formulations using alternative resources to compete with the existing inoculants [22].

In this study we assessed the performance of the carrier formulation consisting of vermiculite as the major component along with cattle manure, wood powder, and wood ash. Thus, we evaluated the ability of this organic carrier formulation to sustain in vitro produced AM fungal propagules so as to colonize the host plant roots.

\section{$2 \quad$ Materials and Methods}

\subsection{AM Fungal Inoculum}

The indigenous AM fungal isolates (Rhizoglomus intraradices and Funneliformis mosseae) obtained from Goa University Arbuscular Mycorrhizal Culture Collection (GUAMCC) were used in the study. These isolates were propagated separately in pot cultures using Plectranthus scutellarioides (L.) R.Br. (coleus) (Lamiaceae) as the host plant. They were grown in soil-sand (1:1) mixture and maintained under controlled green-house conditions $\left(25^{\circ} \mathrm{C}\right.$, RH $\left.80-90 \%\right)$ with no supplementary lighting.

Spores of $R$. intraradices and F. mosseae were extracted from the soil by wet sieving and decanting technique [23]. Isolated spores were then rinsed twice in sterile distilled water and disinfected in 250 $\mu 1$ sodium hypochlorite for 3-5 min. This step was followed by triple rinsing with sterile distilled water and a $10 \mathrm{~min}$ sterilization bath in an antibiotic solution (streptomycin sulfate $0.02 \% \mathrm{w} / \mathrm{v}$ and gentamycin sulfate $0.01 \% \mathrm{w} / \mathrm{v})[7,24]$.

The surface-sterilized spores were plated onto modified Strullu-Romand (MSR) medium [25] for germination, and the Petri plates were incubated in an inverted position in the dark at $27^{\circ} \mathrm{C}$. For the establishment of mycorrhizal association, an actively growing Ri T-DNA transformed root of Cichorium intybus L. or Linum usitatissimum L. with several lateral branches was placed in the vicinity of the germinated spore and incubated in an inverted position in the dark at $27^{\circ} \mathrm{C}$.

For isolation of monoxenically cultured spores of $R$. intraradices and F. mosseae, a small piece of gel containing twenty in vitro produced spores with extra-radical mycelia was cut and added to $25 \mathrm{ml}$ citrate buffer $(0.01 \mathrm{M})$ to dissolve the gel under sterile conditions [26]. The spores along with the attached extra-radical mycelia were then used as inocula.

\subsection{Carrier Preparation}

For formulation of the carrier, initially sterilized sand and vermiculite were used separately as base components to formulate the carrier supplemented with sterilized cow dung powder, wood powder and wood ash in different proportions (Table 1). 
Table 1. Concentrations of carrier formulated in parts (ratios) and percentages with soil and vermiculite separately as base components

\begin{tabular}{c|c|c|c|c|c|c}
\hline Components & Treatment $1^{\mathrm{a}}$ & Treatment $2^{\mathrm{b}}$ & Treatment $3^{\mathrm{a}}$ & Treatment $4^{\mathrm{b}}$ & Treatment $5^{\mathrm{a}}$ & ${\text { Treatment } 6^{\mathrm{b}}}^{--}$ \\
\hline Soil & $\begin{array}{c}20 \\
(60.60 \%)\end{array}$ & -- & $\begin{array}{c}20 \\
(62.50 \%)\end{array}$ & - & $\begin{array}{c}20 \\
(64.51 \%)\end{array}$ & - \\
\hline Vermiculite & -- & $\begin{array}{c}20 \\
(60.60 \%)\end{array}$ & -- & $\begin{array}{c}20 \\
(62.50 \%)\end{array}$ & $\begin{array}{c}20 \\
(64.51 \%)\end{array}$ \\
\hline Cow dung & 8 & 8 & 8 & 8 & 8 & 8 \\
powder & $(24.24 \%)$ & $(24.24 \%)$ & $(25.00 \%)$ & $(25.00 \%)$ & $(25.80 \%)$ & $(25.80 \%)$ \\
\hline Wood powder & 4 & 4 & 3 & 3 & 2 \\
$(12.12 \%)$ & $(12.12 \%)$ & $(9.37 \%)$ & $(9.37 \%)$ & $(6.45 \%)$ & $(6.45 \%)$ \\
\hline Ash & 1 & 1 & 1 & 1 & 1 & 1 \\
$(3.03 \%)$ & $(3.03 \%)$ & $(3.12 \%)$ & $(3.12 \%)$ & $(3.22 \%)$ & $(3.22 \%)$ \\
\hline
\end{tabular}

Note:-- = absent

a Treatments 1, 3, 5 contain soil as the base component

b Treatments 2, 4, 6 contain vermiculite as the base component

It was observed that the treatments containing vermiculite as the base component showed maximum AM colonization as compared to sand (Figure 1), thus vermiculite was selected and used as the base component for the carrier supplemented with sterilized cow dung powder, wood powder, and wood ash in different proportions resulting in 19 formulations/treatments (Table 2). The plant source of wood powder and wood ash was Mangifera indica L. (Anacardiaceae). The carrier materials were sterilized by autoclaving for two consecutive days at $121^{\circ} \mathrm{C}$ for $2 \mathrm{~h}$. The wood powder was washed 3-4 times with tap water followed by drying in the oven and then autoclaving. Physico-chemical characterization of the carrier materials was also carried out, wherein $\mathrm{pH}$ and EC were measured in a 1:1 (v/v) water solution using pH meter (LI 120 Elico, India) and conductivity meter (CM-180 Elico, India). Organic carbon (\%OC) was analyzed by rapid titration method [27]. Available phosphorus (P) was estimated using Bray and Kurtz method [28]. Available potassium (K) was estimated by ammonium acetate method [29] using flame photometer (Systronic 3292). Available micronutrients viz., zinc (Zn), iron (Fe), manganese $(\mathrm{Mn})$ and copper $(\mathrm{Cu})$ were quantified by DTPA-CaCl${ }_{2}$ TEA method [30] using atomic absorption spectrophotometer (AAS 4139). Boron (B) was quantified by the hot water soluble method [31].

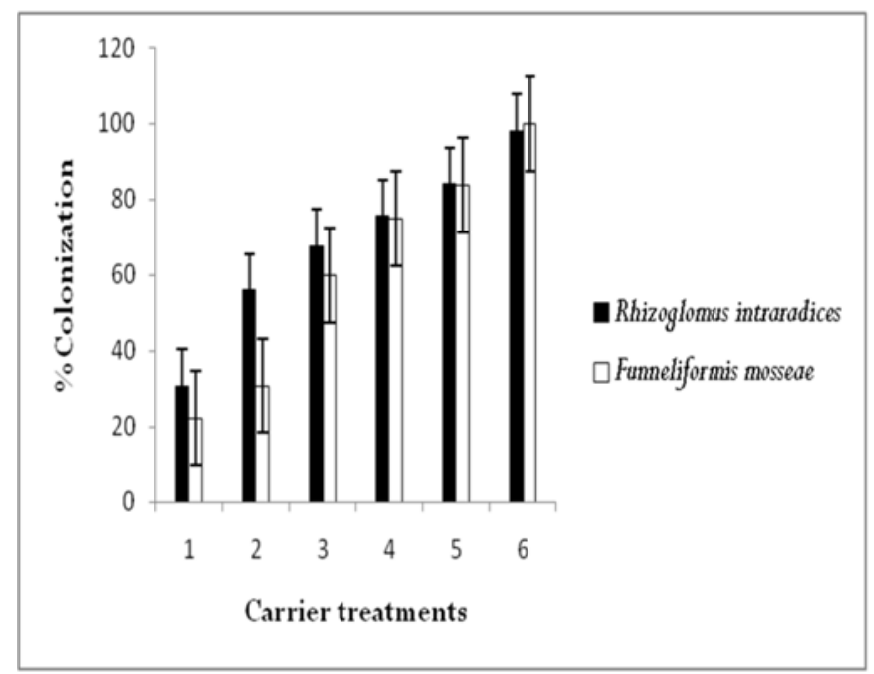

Figure 1. Percent colonization in roots of Eleusine coracana plants inoculated with Rhizoglomus intraradices and Funneliformis mosseae with soil and vermiculite separately as base components. 
Table 2. Carrier formulations in various ratios and percentages

\begin{tabular}{|c|c|c|c|c|}
\hline Treatments & Vermiculite & Cow dung powder & Wood powder & Wood ash \\
\hline 1 & $\begin{array}{c}20 \\
(60.60 \%) \\
\end{array}$ & $\begin{array}{c}8 \\
(24.24 \%) \\
\end{array}$ & $\begin{array}{c}3 \\
(9.09 \%) \\
\end{array}$ & $\begin{array}{c}2 \\
(6.06 \%) \\
\end{array}$ \\
\hline 2 & $\begin{array}{c}20 \\
(74.07 \%) \\
\end{array}$ & $\begin{array}{c}4 \\
(14.81 \%) \\
\end{array}$ & $\begin{array}{c}2 \\
(7.40 \%) \\
\end{array}$ & $\begin{array}{c}1 \\
(3.70 \%) \\
\end{array}$ \\
\hline 3 & $\begin{array}{c}20 \\
(100.00 \%) \\
\end{array}$ & - & - & - \\
\hline 4 & $\begin{array}{c}20 \\
(62.50 \%) \\
\end{array}$ & $\begin{array}{c}8 \\
(25.00 \%) \\
\end{array}$ & $\begin{array}{c}2 \\
(6.25 \%) \\
\end{array}$ & $\begin{array}{c}2 \\
(6.25 \%) \\
\end{array}$ \\
\hline 5 & $\begin{array}{c}20 \\
(64.51 \%) \\
\end{array}$ & $\begin{array}{c}8 \\
(25.80 \%) \\
\end{array}$ & $\begin{array}{c}2 \\
(6.45 \%) \\
\end{array}$ & $\begin{array}{c}1 \\
(3.22 \%) \\
\end{array}$ \\
\hline 6 & $\begin{array}{c}20 \\
(62.50 \%) \\
\end{array}$ & $\begin{array}{c}8 \\
(25.00 \%) \\
\end{array}$ & $\begin{array}{c}3 \\
(9.37 \%) \\
\end{array}$ & $\begin{array}{c}1 \\
(3.12 \%) \\
\end{array}$ \\
\hline 7 & $\begin{array}{c}20 \\
(68.96 \%) \\
\end{array}$ & $\begin{array}{c}4 \\
(13.79 \%) \\
\end{array}$ & $\begin{array}{c}3 \\
(10.34 \%) \\
\end{array}$ & $\begin{array}{c}2 \\
(6.89 \%) \\
\end{array}$ \\
\hline 8 & $\begin{array}{c}20 \\
(71.42 \%) \\
\end{array}$ & $\begin{array}{c}4 \\
(14.28 \%) \\
\end{array}$ & $\begin{array}{c}3 \\
(10.71 \%) \\
\end{array}$ & $\begin{array}{c}1 \\
(3.57 \%) \\
\end{array}$ \\
\hline 9 & $\begin{array}{c}20 \\
(71.42 \%) \\
\end{array}$ & $\begin{array}{c}4 \\
(14.28 \%) \\
\end{array}$ & $\begin{array}{c}2 \\
(7.14 \%) \\
\end{array}$ & $\begin{array}{c}2 \\
(7.14 \%) \\
\end{array}$ \\
\hline 10 & $\begin{array}{c}20 \\
(80.00 \%) \\
\end{array}$ & - & $\begin{array}{c}3 \\
(12.00 \%) \\
\end{array}$ & $\begin{array}{c}2 \\
(8.00 \%) \\
\end{array}$ \\
\hline 11 & $\begin{array}{c}20 \\
(83.33 \%) \\
\end{array}$ & - & $\begin{array}{c}3 \\
(12.50 \%) \\
\end{array}$ & $\begin{array}{c}1 \\
(4.16 \%) \\
\end{array}$ \\
\hline 12 & $\begin{array}{c}20 \\
(83.33 \%) \\
\end{array}$ & - & $\begin{array}{c}2 \\
(8.33 \%) \\
\end{array}$ & $\begin{array}{c}2 \\
(8.33 \%) \\
\end{array}$ \\
\hline 13 & $\begin{array}{c}20 \\
(86.95 \%) \\
\end{array}$ & - & $\begin{array}{c}2 \\
(8.69 \%) \\
\end{array}$ & $\begin{array}{c}1 \\
(4.34 \%) \\
\end{array}$ \\
\hline 14 & $\begin{array}{c}20 \\
(90.90 \%) \\
\end{array}$ & - & - & $\begin{array}{c}2 \\
(9.09 \%) \\
\end{array}$ \\
\hline 15 & $\begin{array}{c}20 \\
(95.23 \%) \\
\end{array}$ & - & - & $\begin{array}{c}1 \\
(4.76 \%) \\
\end{array}$ \\
\hline 16 & $\begin{array}{c}20 \\
(86.95 \%) \\
\end{array}$ & - & $\begin{array}{c}3 \\
(13.04 \%) \\
\end{array}$ & - \\
\hline 17 & $\begin{array}{c}20 \\
(90.90 \%) \\
\end{array}$ & - & $\begin{array}{c}2 \\
(9.09 \%) \\
\end{array}$ & - \\
\hline 18 & $\begin{array}{c}20 \\
(71.42 \%) \\
\end{array}$ & $\begin{array}{c}8 \\
(28.57 \%) \\
\end{array}$ & - & - \\
\hline 19 & $\begin{array}{c}20 \\
(83.33 \%)\end{array}$ & $\begin{array}{c}4 \\
(16.66 \%)\end{array}$ & - & - \\
\hline
\end{tabular}

Note: - : absent

\subsection{Experimental Setup}

The experiment was set up using deep cell plug trays for a period of 3 months. Twenty in vitro produced spores of $R$. intraradices along with colonized transformed chicory (Cichorium intybus L.) roots and F. mosseae spores along with colonized transformed linum (Linum usitatissimum L.) roots were used as inocula in each deep cell plugs containing the carrier formulations and planted with pregerminated seeds of Eleusine coracana Gaertn. (Poaceae) used as host plant. The plants were maintained in the phytotron (Daihan Labtech, LGC-6201G) at 260 lux (16 h photoperiod), $26^{\circ} \mathrm{C}, 41.1 \%$ humidity and 100 ppm $\mathrm{CO}_{2}$ and fertilized with Hoagland's solution [32] minus phosphorus (P) every 20 
days. Six replicates were considered for each treatment. As the replicates were in a single tray, the trays were repositioned at the end of every week.

\subsection{Data Collection and Analysis}

After 3 months of growth, the roots of E. coracana plants were assessed for colonization using Trypan blue staining technique [33]. Various parameters viz, average number of entry points in $1 \mathrm{~cm}$ root segment, root length, total number of infective propagules as per Fertilizer (Control) Order, 1985 [34] and percent colonization [35] were calculated.

\section{$2.5 \quad$ Formulas}

Total number of infection points or infective propagules (IP)= average number of entry points formed in $1 \mathrm{~cm}$ root segment $\times$ total root length (Extrapolate the IP present as numbers per gram of substrate or inoculum)

$\%$ colonization $=$ number of root fragments colonized $\div$ total number of root fragments observed $\times$ 100

\subsection{Statistical Analysis}

The experimental data were subjected to one-way analysis of variance (ANOVA) followed by Tukey post-Hoc pairwise comparison test. Parameters were correlated using Pearson's correlation. Statistical Package for Social Sciences (SPSS) (ver. 22.0 Armonk, NY: IBM Corp.) was used for all statistical analyses.

\section{$3 \quad$ Results}

\subsection{Physico-Chemical Characterization of the Materials Used for Carrier Formulation}

Physico-chemical properties of the carrier materials are depicted in Table 3. It was observed that the materials used for formulation of carrier had different characteristics. Cow dung powder had higher amount of organic carbon (OC) and $\mathrm{P}$, while wood ash had higher potassium (K) content. The micronutrient contents were higher in cow dung powder as compared to wood ash except for copper $(\mathrm{Cu})$. Sterilized vermiculite was used as base component to formulate the carrier supplemented with sterilized cow dung powder, wood powder and wood ash in different proportions. In all, 19 different formulations were prepared by mixing the ingredients with vermiculite in order to review favorable or unfavorable effects of each material in the combination.

Table 3. Physico-chemical parameters of carrier materials

\begin{tabular}{|c|c|c|c|c|c|c|c|c|c|c|}
\hline \multirow[t]{2}{*}{ Carrier material } & \multirow[t]{2}{*}{$\mathrm{pH}$} & \multirow{2}{*}{$\begin{array}{c}\text { E.C. } \\
\text { m.mhos/cm }\end{array}$} & \multicolumn{3}{|c|}{ Macro-nutrients } & \multicolumn{5}{|c|}{ Micro-nutrients (ppm) } \\
\hline & & & $\begin{array}{c}\text { Organic } \\
\text { Carbon* \% }\end{array}$ & $\begin{array}{c}\text { Phosphorus* } \\
\mathrm{Kg} / \mathrm{Ha}\end{array}$ & $\begin{array}{c}\text { Potassium* } \\
\mathrm{Kg} / \mathrm{Ha}\end{array}$ & Zinc* & Iron* & Manganese* & Copper* & Boron* \\
\hline Vermiculite & 7.50 & $<1$ & $\begin{array}{c}0.78 \\
\pm 0.08 \\
\end{array}$ & $\begin{array}{r}10.90 \\
\pm 0.43 \\
\end{array}$ & $\begin{array}{l}170.80 \\
\pm 3.27 \\
\end{array}$ & $\begin{array}{c}0.54 \\
\pm 0.04 \\
\end{array}$ & $\begin{array}{c}1.13 \\
\pm 0.40 \\
\end{array}$ & $\begin{array}{l}17.27 \\
\pm 0.04 \\
\end{array}$ & $\begin{array}{c}0.26 \\
\pm 0.04 \\
\end{array}$ & $\begin{array}{r}1.30 \\
\pm 0.40 \\
\end{array}$ \\
\hline $\begin{array}{c}\text { Cow dung } \\
\text { powder }\end{array}$ & 6.60 & 2.80 & $\begin{array}{c}4.07 \\
\pm 1.13 \\
\end{array}$ & $\begin{array}{c}1038.00 \\
\pm 9.00 \\
\end{array}$ & $\begin{array}{r}2952.00 \\
\pm 93.00 \\
\end{array}$ & $\begin{array}{c}4.41 \\
\pm 0.43 \\
\end{array}$ & $\begin{array}{r}14.44 \\
\pm 0.15 \\
\end{array}$ & $\begin{array}{r}25.54 \\
\pm 0.25 \\
\end{array}$ & $\begin{array}{r}1.25 \\
\pm 0.55 \\
\end{array}$ & $\begin{array}{r}50.60 \\
\pm 0.30 \\
\end{array}$ \\
\hline Wood powder & 5.80 & $<1$ & $\begin{array}{c}1.91 \\
\pm 0.08\end{array}$ & $\begin{array}{r}92.90 \\
\pm 0.99 \\
\end{array}$ & $\begin{array}{l}185.90 \\
\pm 1.10 \\
\end{array}$ & $\begin{array}{c}3.27 \\
\pm 0.07\end{array}$ & $\begin{array}{c}2.84 \\
\pm 0.75 \\
\end{array}$ & $\begin{array}{c}1.91 \\
\pm 0.07\end{array}$ & $\begin{array}{c}0.22 \\
\pm 0.08\end{array}$ & $\begin{array}{l}13.40 \\
\pm 0.30\end{array}$ \\
\hline Wood ash & 10.30 & 12.20 & $\begin{array}{c}0.65 \\
\pm 0.07\end{array}$ & $\begin{array}{l}65.60 \\
\pm 3.80\end{array}$ & $\begin{array}{c}4435.20 \\
\pm 7.10\end{array}$ & $\begin{array}{c}4.10 \\
\pm 0.20\end{array}$ & $\begin{array}{l}10.85 \\
\pm 0.35\end{array}$ & $\begin{array}{c}7.23 \\
\pm 0.02\end{array}$ & $\begin{array}{l}25.00 \\
\pm 0.10\end{array}$ & $\begin{array}{l}25.30 \\
\pm 0.40\end{array}$ \\
\hline
\end{tabular}

*Values are means of three replicates \pm standard deviation. 


\subsection{In Vitro Produced AM fungal Inoculum and Its Germination/Inoculum Potential}

Sporulation in monoxenic cultures of $R$. intraradices was observed 18-20 days after association with transformed chicory roots and continued up to 7 months. In monoxenic cultures of $F$. mosseae, sporulation was initiated 15-20 days after association with transformed linum roots and continued up to 7 months. It was observed that in vitro produced spores of both AM species showed maximum germination potential at 28 weeks (i.e. fully matured spores at 197 days) that exhibited maximum germination when placed on MSR medium and hence were selected for preparation of inocula.

\subsection{Effect of Different Carrier Treatments on Re-inoculation/Colonization Potential of In Vitro Produced AM Fungal Inoculum}

The in vitro produced spores of $R$. intraradices and $F$. mosseae along with the attached extra-radical mycelia were used separately as inocula to colonize E. coracana plants. For $R$. intraradices, maximum number of entry points (13) per root segment in E. coracana were recorded in treatment 5 while for $F$. mosseae, the maximum number of entry points (7.33) per root segment in the same host plant were also recorded in the same treatment (Table 4). Similarly, the total number of infective propagules was highest (148 infection points/g of inoculum used) for $R$. intraradices in treatment 5 while for F. mosseae, it was highest (127 infection points/g of inoculum used) in the same treatment (Table 4). Percent colonization was highest (100\%) in treatment 5 for both the AM species (Table 4). Treatment 5 was found to be optimum for both the species.

Table 4. Average number of entry points, average root length, infectivity potential and percent colonization in roots of E. coracana plants inoculated with R. intraradices and F. mosseae

\begin{tabular}{|c|c|c|c|c|c|c|c|c|}
\hline \multirow[t]{2}{*}{ Treatment } & \multicolumn{2}{|c|}{$\begin{array}{l}\text { Average number of entry } \\
\text { points* }\end{array}$} & \multicolumn{2}{|c|}{$\begin{array}{l}\text { Average root length* } \\
\qquad(\mathrm{cm})\end{array}$} & \multicolumn{2}{|c|}{$\begin{array}{c}\text { Total number of infective } \\
\text { propagules* (IP) } \\
\left(\mathrm{g}^{-1} \text { of inoculum) }\right.\end{array}$} & \multicolumn{2}{|c|}{$\begin{array}{c}\text { Percent colonization* } \\
(\%)\end{array}$} \\
\hline & $\begin{array}{c}R . \\
\text { intraradices }\end{array}$ & F. mosseae & $\begin{array}{c}R . \\
\text { intraradices }\end{array}$ & F. mosseae & R. intraradices & F. mosseae & $\begin{array}{c}R . \\
\text { intraradices }\end{array}$ & F. mosseae \\
\hline 1 & $\begin{array}{c}6.00 \mathrm{de} \pm \\
0.00\end{array}$ & $\begin{array}{c}5.50 \mathrm{~cd} \pm \\
0.14 \\
\end{array}$ & $2.38 \mathrm{a} \pm 1.02$ & $\begin{array}{c}2.69 \mathrm{a} \pm \\
0.62 \\
\end{array}$ & 85.68 de \pm 3.78 & 88.77 ef \pm 3.34 & $\begin{array}{c}80.71 \mathrm{de} \pm \\
5.81\end{array}$ & $\begin{array}{c}80.20 \mathrm{e} \pm \\
4.56\end{array}$ \\
\hline 2 & $\begin{array}{c}5.33 \text { efg } \pm \\
0.51\end{array}$ & $\begin{array}{c}5.60 \mathrm{~cd} \pm \\
0.13\end{array}$ & $3.06 \mathrm{a} \pm 0.62$ & $\begin{array}{c}2.68 \mathrm{a} \pm \\
0.61\end{array}$ & $97.85 \mathrm{c} \pm 9.03$ & $\begin{array}{c}90.04 \text { def } \pm \\
4.44\end{array}$ & $\begin{array}{c}86.57 \mathrm{c} \pm \\
7.06\end{array}$ & $\begin{array}{c}85.15 \mathrm{~cd} \pm \\
3.49\end{array}$ \\
\hline 3 & $6.50 \mathrm{~d} \pm 0.54$ & $\begin{array}{c}4.38 \mathrm{fg} \pm \\
0.32\end{array}$ & $1.95 \mathrm{a} \pm 0.82$ & $\begin{array}{c}2.70 \mathrm{a} \pm \\
0.80\end{array}$ & $76.05 \mathrm{fgh} \pm 8.00$ & $70.95 \mathrm{hi} \pm 8.00$ & $\begin{array}{c}70.00 \mathrm{~g} \pm \\
5.51\end{array}$ & $\begin{array}{c}62.48 \mathrm{gh} \pm \\
4.25\end{array}$ \\
\hline 4 & $6.50 \mathrm{~d} \pm 0.54$ & $\begin{array}{c}5.60 \mathrm{~cd} \pm \\
0.08 \\
\end{array}$ & $2.43 \mathrm{a} \pm 1.15$ & $\begin{array}{c}3.03 \mathrm{a} \pm \\
0.59 \\
\end{array}$ & $95.16 \mathrm{~cd} \pm 9.04$ & $\begin{array}{c}101.80 \mathrm{bc} \pm \\
4.07 \\
\end{array}$ & $\begin{array}{c}90.00 \mathrm{bc} \pm \\
6.32 \\
\end{array}$ & $\begin{array}{c}82.85 \text { cde } \pm \\
6.92 \\
\end{array}$ \\
\hline 5 & $\begin{array}{c}13.00 \mathrm{a} \pm \\
0.00\end{array}$ & $\begin{array}{c}7.38 \mathrm{a} \pm \\
0.13 \\
\end{array}$ & $1.90 \mathrm{a} \pm 0.71$ & $\begin{array}{c}2.86 \mathrm{a} \pm \\
0.57 \\
\end{array}$ & $148.20 \mathrm{a} \pm 9.11$ & $\begin{array}{c}126.64 \mathrm{a} \pm \\
10.88 \\
\end{array}$ & $\begin{array}{c}100.00 \mathrm{a} \pm \\
0.00 \\
\end{array}$ & $\begin{array}{c}100.00 \mathrm{a} \pm \\
0.00 \\
\end{array}$ \\
\hline 6 & $\begin{array}{c}5.00 \mathrm{fgh} \pm \\
0.00 \\
\end{array}$ & $\begin{array}{c}6.05 \mathrm{bc} \pm \\
0.49 \\
\end{array}$ & $2.79 \mathrm{a} \pm 0.74$ & $\begin{array}{c}2.45 \mathrm{a} \pm \\
1.11 \\
\end{array}$ & 83.70 ef \pm 7.60 & 88.93 ef \pm 6.26 & $\begin{array}{c}78.42 \mathrm{e} \pm \\
7.13 \\
\end{array}$ & $\begin{array}{c}81.86 \text { de } \pm \\
3.50 \\
\end{array}$ \\
\hline 7 & $9.00 \mathrm{c} \pm 0.00$ & $\begin{array}{c}7.05 \mathrm{a} \pm \\
0.49\end{array}$ & $1.83 \mathrm{a} \pm 0.78$ & $\begin{array}{c}2.29 \mathrm{a} \pm \\
0.94\end{array}$ & $98.82 \mathrm{c} \pm 7.77$ & $96.86 \mathrm{~cd} \pm 8.19$ & $\begin{array}{c}90.57 \mathrm{bc} \pm \\
4.54\end{array}$ & $\begin{array}{c}87.44 \mathrm{c} \pm \\
6.30\end{array}$ \\
\hline 8 & $\begin{array}{c}5.33 \text { efg } \pm \\
0.51 \\
\end{array}$ & $\begin{array}{c}5.60 \mathrm{~cd} \pm \\
0.08 \\
\end{array}$ & $2.93 \mathrm{a} \pm 0.53$ & $\begin{array}{c}2.85 \mathrm{a} \pm \\
0.50 \\
\end{array}$ & $93.70 \mathrm{~cd} \pm 5.79$ & $\begin{array}{c}95.76 \text { cde } \pm \\
7.11 \\
\end{array}$ & $\begin{array}{c}85.28 \mathrm{~cd} \pm \\
8.92 \\
\end{array}$ & $\begin{array}{c}85.15 \mathrm{~cd} \pm \\
3.49 \\
\end{array}$ \\
\hline 9 & $\begin{array}{c}11.00 \mathrm{~b} \pm \\
1.89 \\
\end{array}$ & $\begin{array}{c}7.05 \mathrm{a} \pm \\
0.49 \\
\end{array}$ & $1.98 \mathrm{a} \pm 0.81$ & $\begin{array}{c}2.48 \mathrm{a} \pm \\
0.98 \\
\end{array}$ & $130.68 \mathrm{~b} \pm 22.86$ & $104.90 \mathrm{~b} \pm 8.02$ & $\begin{array}{c}93.33 \mathrm{~b} \pm \\
6.06 \\
\end{array}$ & $\begin{array}{c}94.76 \mathrm{~b} \pm \\
1.42 \\
\end{array}$ \\
\hline 10 & $6.50 \mathrm{~d} \pm 1.64$ & $\begin{array}{c}5.05 \text { de } \pm \\
0.77 \\
\end{array}$ & $1.97 \mathrm{a} \pm 0.81$ & $\begin{array}{c}2.57 \mathrm{a} \pm \\
0.81 \\
\end{array}$ & 76.83 efg \pm 8.30 & $77.87 \mathrm{gh} \pm 6.77$ & $\begin{array}{c}66.06 \mathrm{gh} \pm \\
4.92 \\
\end{array}$ & $\begin{array}{c}66.35 \mathrm{fg} \pm \\
5.57 \\
\end{array}$ \\
\hline 11 & $\begin{array}{l}6.00 \mathrm{de} \pm \\
1.09\end{array}$ & $\begin{array}{c}4.63 \text { ef } \pm \\
0.49\end{array}$ & $2.16 \mathrm{a} \pm 0.91$ & $\begin{array}{c}2.80 \mathrm{a} \pm \\
0.48\end{array}$ & 77.76 efg \pm 5.45 & $77.78 \mathrm{~g} \pm 5.34$ & $\begin{array}{c}70.63 \mathrm{~g} \pm \\
5.57\end{array}$ & $\begin{array}{c}67.53 \mathrm{f} \pm \\
4.74\end{array}$ \\
\hline
\end{tabular}




\begin{tabular}{|c|c|c|c|c|c|c|c|c|}
\hline 12 & $\begin{array}{c}4.33 \mathrm{hi} \pm \\
0.51 \\
\end{array}$ & $\begin{array}{c}5.33 \mathrm{~d} \pm \\
0.18 \\
\end{array}$ & $2.58 \mathrm{a} \pm 0.90$ & $\begin{array}{c}2.71 \mathrm{a} \pm \\
0.42 \\
\end{array}$ & $67.02 \mathrm{~h} \pm 6.64$ & $86.66 \mathrm{f} \pm 4.97$ & $\begin{array}{c}62.31 \mathrm{~h} \pm \\
7.10 \\
\end{array}$ & $\begin{array}{c}67.77 \mathrm{f} \pm \\
5.44 \\
\end{array}$ \\
\hline 13 & $\begin{array}{c}4.66 \text { ghi } \pm \\
0.51 \\
\end{array}$ & $\begin{array}{c}4.05 \mathrm{~g} \pm \\
0.49 \\
\end{array}$ & $2.41 \mathrm{a} \pm 1.06$ & $\begin{array}{c}2.80 \mathrm{a} \pm \\
0.56 \\
\end{array}$ & $67.38 \mathrm{~h} \pm 7.03$ & $68.04 \mathrm{ij} \pm 4.49$ & $\begin{array}{c}62.66 \mathrm{~h} \pm \\
4.84 \\
\end{array}$ & $\begin{array}{c}61.42 \mathrm{~h} \pm \\
5.35 \\
\end{array}$ \\
\hline 14 & $2.00 \mathrm{j} \pm 1.09$ & $\begin{array}{c}2.05 \mathrm{~h} \pm \\
1.02\end{array}$ & $1.88 \mathrm{a} \pm 0.72$ & $\begin{array}{c}1.70 \mathrm{a} \pm \\
0.77\end{array}$ & $22.56 \mathrm{j} \pm 1.63$ & $20.911 \pm 5.07$ & $\begin{array}{c}14.18 \mathrm{j} \pm \\
2.26\end{array}$ & $\begin{array}{c}9.97 \mathrm{k} \pm \\
6.16\end{array}$ \\
\hline 15 & $2.00 \mathrm{j} \pm 0.89$ & $\begin{array}{c}2.08 \mathrm{~h} \pm \\
0.59 \\
\end{array}$ & $2.00 \mathrm{a} \pm 1.03$ & $\begin{array}{c}1.99 \mathrm{a} \pm \\
1.03 \\
\end{array}$ & $24.00 \mathrm{j} \pm 6.08$ & $24.831 \pm 6.33$ & $\begin{array}{c}11.64 \mathrm{j} \pm \\
3.62 \\
\end{array}$ & $\begin{array}{c}14.77 \mathrm{j} \pm \\
4.74 \\
\end{array}$ \\
\hline 16 & $\begin{array}{c}5.00 \mathrm{fgh} \pm \\
0.00 \\
\end{array}$ & $\begin{array}{c}4.05 \mathrm{~g} \pm \\
0.64 \\
\end{array}$ & $2.33 \mathrm{a} \pm 0.97$ & $\begin{array}{c}1.96 \mathrm{a} \pm \\
1.07 \\
\end{array}$ & $69.90 \mathrm{gh} \pm 3.11$ & $47.62 \mathrm{k} \pm 3.35$ & $\begin{array}{c}60.78 \mathrm{hi} \pm \\
6.76 \\
\end{array}$ & $\begin{array}{c}59.50 \mathrm{~h} \pm \\
3.87 \\
\end{array}$ \\
\hline 17 & $4.00 \mathrm{i} \pm 0.63$ & $\begin{array}{c}5.05 \text { de } \pm \\
0.49 \\
\end{array}$ & $2.38 \mathrm{a} \pm 1.02$ & $\begin{array}{c}2.01 \mathrm{a} \pm \\
1.01 \\
\end{array}$ & $57.12 \mathrm{i} \pm 9.37$ & $60.90 \mathrm{j} \pm 3.29$ & $\begin{array}{c}56.74 \mathrm{i} \pm \\
3.27 \\
\end{array}$ & $\begin{array}{c}53.48 \mathrm{i} \pm \\
3.79 \\
\end{array}$ \\
\hline 18 & $\begin{array}{c}5.66 \text { def } \pm \\
1.03 \\
\end{array}$ & $\begin{array}{c}6.38 \mathrm{~b} \pm \\
0.44 \\
\end{array}$ & $2.41 \mathrm{a} \pm 1.20$ & $\begin{array}{c}1.85 \mathrm{a} \pm \\
0.91 \\
\end{array}$ & 81.80 ef \pm 6.33 & $70.81 \mathrm{i} \pm 4.67$ & $\begin{array}{c}76.58 \text { ef } \pm \\
4.01\end{array}$ & $\begin{array}{c}60.41 \mathrm{~h} \pm \\
5.10 \\
\end{array}$ \\
\hline 19 & $\begin{array}{c}4.66 \text { ghi } \pm \\
0.51 \\
\end{array}$ & $\begin{array}{c}6.05 \mathrm{bc} \pm \\
0.64\end{array}$ & $2.79 \mathrm{a} \pm 0.54$ & $\begin{array}{c}1.84 \mathrm{a} \pm \\
0.92 \\
\end{array}$ & 78.00 efg \pm 5.00 & $66.79 \mathrm{ij} \pm 4.69$ & $\begin{array}{c}71.53 \mathrm{fg} \pm \\
5.55\end{array}$ & $\begin{array}{c}58.09 \mathrm{hi} \pm \\
4.67 \\
\end{array}$ \\
\hline
\end{tabular}

*Values are means of six replicates \pm standard deviation. Values in the same column not sharing the same letters are significantly different $(P \leqslant 0.05)$

As treatment 5 was observed to be the optimum for both AM species, physico-chemical parameters of treatment 5 were analyzed. Physico-chemical parameters of treatment 14 and 15 were also analyzed which showed least AM fungal infection on the whole (Table 5).

Table 5. Physico-chemical parameters of carrier formulations (treatments 5, 14, 15)

\begin{tabular}{|c|c|c|c|c|c|c|c|c|c|c|}
\hline \multirow[t]{2}{*}{ Treatments } & \multirow[t]{2}{*}{$\mathrm{pH}$} & \multirow{2}{*}{$\begin{array}{c}\text { E.C. } \\
\text { m.mhos } / \mathrm{cm}\end{array}$} & \multicolumn{3}{|c|}{ Macro-nutrients } & \multicolumn{5}{|c|}{ Micro-nutrients (ppm) } \\
\hline & & & $\begin{array}{c}\text { Organic } \\
\text { Carbon\% }\end{array}$ & $\begin{array}{c}\text { Phosphorus } \\
\mathrm{Kg} / \mathrm{Ha}\end{array}$ & $\begin{array}{c}\text { Potassium } \\
\mathrm{Kg} / \mathrm{Ha}\end{array}$ & Zinc & Iron & Manganese & Copper & Boron \\
\hline 5 & 8.20 & 1.70 & 2.55 & 371.70 & 2360.00 & 3.75 & 7.44 & 20.74 & 1.70 & 5.30 \\
\hline 14 & 9.60 & 1.20 & 0.30 & 229.90 & 3946.00 & 58.40 & 1.74 & 153.70 & 303.90 & 30.42 \\
\hline 15 & 9.20 & 0.60 & 0.32 & 130.40 & 3472.00 & 8.17 & 3.00 & 101.60 & 15.62 & 8.79 \\
\hline
\end{tabular}

Analysis of variance was calculated to compare the effect of the carrier treatments on percent colonization by AM fungal species. Analysis of variance revealed that the effect of carrier treatment on percent colonization by both the AM species was significant, $F(18,95)=106.090, p \leqslant 0.05$ for $R$. intraradices and $F(18,95)=152.678, p \leqslant 0.05$ for $F$. mosseae (Table 6).

Table 6. Analysis of variance for percent colonization

\begin{tabular}{c|c|c|c|r|r|r|r|r|r}
\hline & & \multicolumn{5}{|c|}{$R$. intraradices } & \multicolumn{5}{c}{$F$. mosseae } \\
\hline Source & $d f^{*}$ & $S S^{*}$ & $M S^{*}$ & $F$ & $P$ & $S S^{*}$ & $M S^{*}$ & $F$ & $P$ \\
\hline Between & 18 & 59503.949 & 3305.775 & 106.090 & $\leqslant 0.05$ & 59931.998 & 3329.555 & 152.678 & $\leqslant 0.05$ \\
\hline Within & 95 & 2960.222 & 31.160 & & & 2071.726 & 21.808 & & \\
\hline Total & 113 & 62464.171 & & & & 62003.724 & & & \\
\hline
\end{tabular}

$* d f$ degrees of freedom; $S S$ sum of squares; $M S$ mean square

A Pearson product-moment correlation coefficient was computed to assess the relationship between the infective propagules and percent colonization by both the AM fungal species. There was a positive correlation between the two variables $[\mathrm{r}=0.926, \mathrm{n}=19, p \leqslant 0.01]$ for $R$. intraradices and $[\mathrm{r}=0.978, \mathrm{n}$ $=19, p \leqslant 0.01]$ for $F$. mosseae. Overall, a strong positive correlation between the infective propagules and percent colonization was observed (Figure 2; Figure 3). 


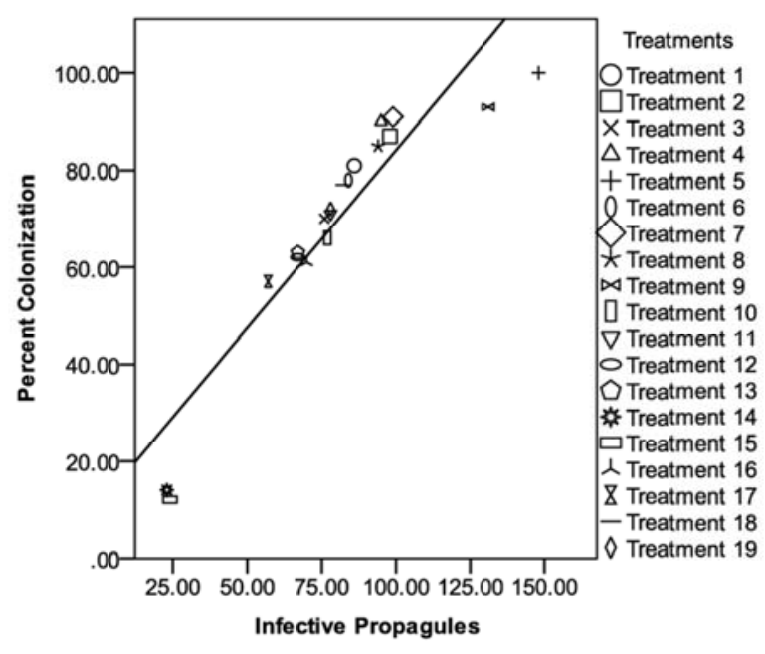

Figure 2. Correlation between infective propagules and percent colonization by Rhizoglomus intraradices.

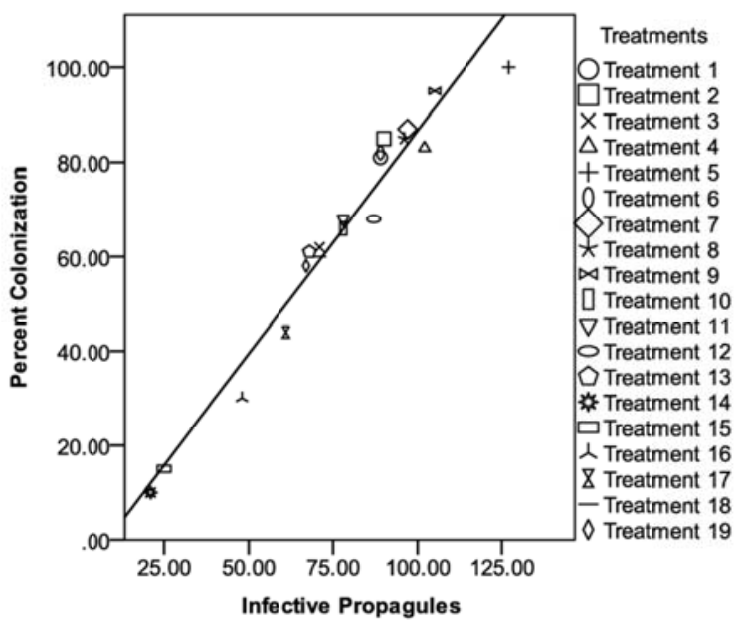

Figure 3. Correlation between infective propagules and percent colonization by Funneliformis mosseae.

\section{Discussions}

Several researchers have proposed different methods for production of AM fungal inocula in soil based cultures as well as carrier based inocula [36]. Soil-less techniques, such as monoxenic culture have advantage of being less bulky, less sensitive to contamination, more concentrated and more uniform than soil cultures, and the propagules can be easily harvested [37, 19]. More recently, methods based on transformed root organ cultures feasible for implementation on a commercial scale have been developed that allow production of high concentration of propagules in a limited space [38-40]. But commercially, mycorrhizal inoculum is still produced mainly via the conventional method where host plants are grown under controlled conditions using sand/soil as the substrate for mass production of the inoculum in pots, bags, or beds for large-scale application.

Present study represents the first attempt to develop a suitable carrier formulation comprising of vermiculite, cow dung powder, wood powder and wood ash for mass multiplication of in vitro produced AM fungal inocula. Both the in vitro produced AM fungal species ( $R$. intraradices and F. mosseae) responded more positively to treatment 5 comprising of carrier formulation (vermiculite: cow dung powder: wood powder: wood ash) in the ratio of 20:8:2:1 through $100 \%$ colonization highlighting the positive interaction between AM, carrier formulation and host plant. Organic amendments are rich in nutrients and their positive influence on AM fungal root colonization has been reported earlier [41-43, 8, 
44, 45]. Addition of organic residues to the substrate is known to increase AM fungal sporulation hence leading to increased inoculum production [46-49]. Douds et al. [8] successfully produced AM fungal inoculum in compost mixed with vermiculite, perlite, or horticultural potting media and observed that the propagule numbers were maximum in vermiculite based media. They reported that the laminar sheets of vermiculite create favorable conditions for growth and persistence of AM fungal hyphae since similar spore populations and colonization of roots among the three media amendments were observed. The carrier formulation developed in our study offers several other benefits in addition to maintaining the inoculum potential of in vitro produced AM fungal propagules. The carrier materials with the exception of vermiculite are organic in nature besides providing macro- and micro-nutrients, increase substrate permeability and improve water retention.

Physico-chemical characterization of treatment 5 revealed that although the treatment had high concentration of nutrients especially $\mathrm{P}$, it did not affect AM colonization. Bolan and Robson [50] reported significant effects of increased $\mathrm{P}$ supply resulting in increased formation of mycorrhizal structures. Addition of $\mathrm{P}$ increased both root growth and the percentage of root length colonized by AM fungi. If AM fungal isolates are produced in organic substrate with high $\mathrm{P}$ levels it is likely that the isolates will be more adapted to conditions of high $\mathrm{P}$ [51, 49]. However, the overall least AM fungal interaction was observed in treatments 14 and 15 . This may be attributed to the absence of cow dung and wood powder in the treatments. The physico-chemical characterization of treatments 14 and 15 revealed high levels of $\mathrm{K}, \mathrm{Zn}, \mathrm{Mn}, \mathrm{Cu}$ and $\mathrm{B}$ as compared to the optimum carrier formulation (treatment 5). High concentrations of $\mathrm{Zn}, \mathrm{Mn}, \mathrm{Cu}, \mathrm{B}$ and $\mathrm{K}$ suppress spore germination, root colonization and mycelial growth of AM fungi [52-56].

In the present study, a strong positive correlation between the infective propagules and percent colonization was observed. The importance of entry points for the development of mycorrhizal structures within the roots and ensuing overall effectiveness of AM fungi is well known [57]. After spore germination, the AM fungal hyphae grows towards the host plant roots $[58,59]$, followed by penetration into the root cortical cells and leading to formation of intra-radical structures. Scervino et al. [60,61] reported a close relationship between the number of entry points and the degree of colonization.

\section{Conclusion}

Both the isolates used in the present investigation were highly infective and efficient in stimulating colonization and sporulation when re-inoculated with the carrier formulation. This study reports the successful formulation of AM inocula using organic based carrier materials. Such an attempt indicates a strong possibility for enhancing plant growth and productivity. Further studies in this direction are in progress.

Acknowledgements. The first author gratefully acknowledges the financial assistance received from Innovation in Science Pursuit For Inspired Research (INSPIRE) programme, Department of Science and Technology (DST), Government of India, New Delhi under Grant Dy. No. C/3236/IFD/2014-15 to carry out this study.

\section{References}

1. D. Owen, A. P. Williams, G. W. Griffith, and P. J. A. Withers, "Use of commercial bio-inoculants to increase agricultural production through improved phosphorus acquisition," Applied Soil Ecology, vol. 86, pp. 41-54, 2015.

2. S. E. Smith and D. J. Read, Mycorrhizal Symbiosis. Academic Press, London, 2008.

3. M. W. Schwartz, J. D. Hoeksema, C. A. Gehring, N. C. Johnson, J. N. Klironomos, L. K. Abbott, and A. Pringle, "The promise and the potential consequences of the global transport of mycorrhizal fungal inoculum," Ecology Letters, vol. 9, pp. 501-515, 2006.

4. D. D. Douds, V. Gadkar, and A. Adholeya, "Mass production of VAM fungus biofertilizer," in Mycorrhizal Biology. Kluwer Academic Press, New York, 2000, pp. 197-215.

5. S. Singh, "Mass production of AM fungi: Part 1," Mycorrhiza News, vol. 14, pp. 2-9, 2002. 
6. S. Gianinazzi and M. Vosátka, "Inoculum of arbuscular mycorrhizal fungi for production systems: science meets business," Canadian Journal of Botany, vol. 82, pp. 1264-1271, 2004.

7. G. Bécard and J. A. Fortin, "Early events of vesicular-arbuscular mycorrhiza formation on Ri T-DNA transformed roots," New Phytologist, vol. 108, pp. 211-218, 1988.

8. D. D. Douds, G. Nagahashi, and P. R. Hepperly, "On-farm production of inoculum of indigenous arbuscular mycorrhizal fungi and assessment of diluents of compost for inoculum production," Bioresource Technology, vol. 101, pp. 2326-2330, 2010.

9. C. Accinelli, S. M. Ludovica, H. K. Abbas, R. M. Zablotowicz, and J. R. Wilkinson, "Use of a granular bioplastic formulation for carrying conidia of a non-aflatoxigenic strain of Aspergillus flavus," Bioresource Technology, vol. 100, pp. 3997-4004, 2009.

10.G. P. Brahmaprakash and P. K. Sahu, "Biofertilizers for Sustainability," Journal of Indian Institute of Science, vol. 92, pp. 37-62, 2012.

11.J. H. G. Stephens and H. M. Rask, "Inoculant production and formulation," Field Crops Research, vol. 65, pp. 249-258, 2000.

12.F. B. Rebah, R. D. Tyagi, and D. Prevost, "Wastewater sludge as a substrate for growth and carrier for rhizobia: the effect of storage conditions on survival of Sinorhizobium meliloti," Bioresource Technology, vol. 83, no. 2, pp. $145-151,2002$.

13.M. C. Rivera-Cruz, A. T. Narcía, G. C. Ballona, J. Kohler, F. Caravaca, and A. Roldán, "Poultry manure and banana waste are effective biofertilizer carriers for promoting plant growth and soil sustainability in banana crops," Soil Biology and Biochemistry, vol. 40, no. 12, pp. 3092-3095, 2008.

14.B. C. Mallesha, D. J. Bagyaraj, and G. Pai, "Perlite-soilrite mix as a carrier for mycorrhiza and rhizobia to inoculate Leucaena leucocephala," Leucaena Research Reports, vol. 13, pp. 32-33, 1992.

15.D. Redecker, H. Thierfelder, and D. Werner, "A new cultivation system for arbuscular mycorrhizal fungi on glass beads," Angewandte Botanik, vol. 69, pp. 189-191, 1995.

16.Y. Bashan, "Inoculants of plant growth-promoting bacteria for use in agriculture," Biotechnology Advances, vol. 16, no. 4, pp. 729-770, 1998.

17.A. Gaur and A. Adholeya, "Effects of the particle of soil-less substrates upon AM fungus inoculum production," Mycorrhiza, vol. 10, no. 1, pp. 43-48, 2000.

18.D. F. Herridge, M. B. Peoples, and R. M. Boddey, "Global inputs of biological nitrogen fixation in agricultural systems," Plant and Soil, vol. 311, no. 1, pp. 1-18, 2008.

19.E. Malusá, L. Sas-Paszt, and J. Ciesielska, "Technologies for beneficial microorganisms inocula used as biofertilizers," Scientific World Journal, pp. 2012:491206, 2012.

20.L. Herrmann and D. Lesueur, "Challenges of formulation and quality of biofertilizers for successful inoculation," Applied Microbiology and Biotechnology, vol. 97, no. 20, pp. 8859-8873, 2013.

21.H. Wang, L. Shen, L. Zhai, J. Zhang, T. Ren, B. Fan, and H. Liu, "Preparation and utilization of phosphate biofertilizers using agricultural waste," Journal of Integrative Agriculture, vol. 14, no. 1, pp. 158-167, 2015.

22.R. P. John, R. D. Tyagi, S. K. Brar, R. Y. Surampalli, and D. Prevost, "Bio-encapsulation of microbial cells for targeted agricultural delivery," Critical Reviews in Biotechnology, vol. 31, no. 3, pp. 211-226, 2011.

23.J. W. Gerdemann and T. H. Nicolson, "Spores of mycorrhizal Endogone species extracted from soil by wet sieving and decanting," Transactions of the British Mycological Society, vol. 46, no. 2, pp. 235-244, 1963.

24.B. Mosse, "The regular germination of resting spores and some observations on the growth requirements of an Endogone sp. causing vesicular-arbuscular mycorrhiza," Transactions of the British Mycological Society, vol. 42, no. 3, pp. 273-286, 1959.

25.S. Declerck, D. G. Strullu, and C. Plenchette, "Monoxenic culture of the intraradical forms of Glomus sp. isolated from a tropical ecosystem: a proposed methodology for germplasm collection," Mycologia, vol. 90, no. 4, pp. 579-585, 1998.

26.S. Cranenbrouck, L. Voets, C. Bivort, L. Renard, D. G. Strullu, and S. Declerck, "Methodologies for in vitro cultivation of arbuscular mycorrhizal fungi with root-organs," in In vitro culture of mycorrhizas. Springer-Verlag, Heidelberg, 2005, pp. 341-375.

27.A. Walkley and J. A. Black, "An examination of the Degtjareff method for determining soil organic matter and a proposed modification of the chromic titration method," Soil Science, vol. 37, no. 1, pp. 29-38, 1934. 
28.R. H. Bray and L. T. Kurtz, "Determination of total, organic and available forms of phosphorus in soils," Soil Science, vol. 59, no. 1, pp. 39-46, 1945.

29.J. J. Hanway and H. Heidel, "Soil analysis method as used in Iowa State College Soil Testing Laboratory," Iowa Agriculture, vol. 57, pp. 1-31, 1952.

30.W. L. Lindsay and W. A. Norvell, "Development of DTPA soil test for zinc, iron, manganese and copper," Soil Science Society of America Journal, vol. 42, no. 3, pp. 421-428, 1978.

31.K. C. Berger and E. Truog, "Boron determination in soils and plants," Industrial and Engineering Chemistry Analytical Edition, vol. 11, no. 10, pp. 540-545, 1939.

32.D. R. Hoagland and D. I. Arnon, "The water-culture method for growing plants without soil. Berkeley, Calif. : University of California, College of Agriculture, Agricultural Experiment Station," Circular 347, 1950.

33.J. M. Phillips and D. S. Hayman, "Improved procedures for clearing roots and staining parasitic and vesiculararbuscular mycorrhizal fungi for rapid assessment of infection," Transactions of the British Mycological Society, vol. 55, no. 1, pp. 158-161, 1970.

34.Ministry of Agriculture, Government of India, "Biofertilizers and organic fertilizers covered in fertilizer (Control) Order, 1985 (as amended, March 2006 and November 2009)," Official Gazette 3 November, 2009.

35.D. J. Read, H. K. Koucheki, and J. Hodgson, "Vesicular-arbuscular mycorrhiza in natural vegetation systems. I. The occurrence of infection," New Phytologist, vol. 77, no. 3, pp. 641-653, 1976.

36.A. Adholeya, "Commercial production of AMF through industrial mode and its large scale application." Proceedings of the 4th international conference Mycorrhizae (ICOM4), Montreal, Canada, 2003.

37.Y. Okon and H. Hadar, "Microbial inoculants as crop-yield enhancers," Critical Reviews in Biotechnology, vol. 6, no. 1 , pp. 61-85, 1987.

38.M. St-Arnaud, C. Hamel, B. Vimard, M. Caron, and J. A. Fortin, "Enhanced hyphal growth and spore production of the arbuscular mycorrhizal fungus Glomus intraradices in an in vitro system in the absence of host roots," Mycological Research, vol. 100, no. 3, pp. 328-332, 1996.

39.Y. Dalpé and M. Monreal, "Arbuscular mycorrhiza inoculum to support sustainable cropping systems," Online Crop Management 3, 2004.

40.A. Adholeya, P. Tiwari, and R. Singh, "Large-scale inoculum production of arbuscular mycorrhizal fungi on root organs and inoculation strategies," in In vitro culture of mycorrhizas. Springer, Heidelberg, 2005, pp. 315-338.

41.A. Gaur and A. Adholeya, "Arbuscular-mycorrhizal inoculation of five tropical fodder crops and inoculum production in marginal soil amended with organic matter," Biology and Fertility of Soils, vol. 35, no. 3, pp. 214$218,2002$.

42.M. Gryndler, H. Hrselová, R. Sudová, H. Gryndlerová, V. Rezácová, and V. Merhautová, "Hyphal growth and mycorrhiza formation by the arbuscular fungus Glomus claroideum BEG23 is stimulated by humic substances," Mycorrhiza, vol. 15, no. 7, pp. 483-488, 2005.

43.H. Perner, D. Schwarz, and E. George, "Effects of mycorrhizal inoculation and compost supply on growth and nutrient uptake of young leek plants grown in peat-based substrates," HortScience, vol. 41, no. 3, pp. 628-632, 2006.

44.K. Saranya and K. Kumutha, "Standardization of the substrate material for large scale production of arbuscular mycorrhizal inoculum," International Journal of Agriculture Sciences, vol. 3, no. 1, pp. 71-77, 2011.

45.A. Tanwar, A. Aggarwal, A. Yadav, and V. Parkash, "Screening and selection of efficient host and sugarcane bagasse as substrate for mass multiplication of Funneliformis mosseae," Biological Agriculture and Horticulture, vol. 29, no. 2, pp. 107-117, 2013.

46.A. Gaur and A. Adholeya, "Diverse response of five ornamental plant species to mixed indigenous and single isolate arbuscular mycorrhizal inocula in marginal soil amended with organic matter," Journal of plant nutrition, vol. 28, no. 4, pp. 707-723, 2005.

47.F. S. B. Silva, A. M. Yano-Melo, J. A. C. Brandão, and L. C. Maia, "Sporulation of arbuscular mycorrhizal fungi using Tris-HCl buffer in addition to nutrient solutions," Brazilian Journal of Microbiology, vol. 36, no. 4, pp. 327-332, 2005.

48.D. D. Jr. Douds, G. Nagahashi, P. E. Pfeffer, C. Reider, and W. M. Kayser, "On-farm production of AM fungus inoculum in mixture of compost and vermiculite," Bioresource Technology, vol. 97, no. 6, pp. 809-818, 2006. 
49.I. R. Coelho, M. V. L. Pedone-Bonfim, F. S. B. Silva, and L. C. Maia, "Optimization of the production of mycorrhizal inoculum on substrate with organic fertilizer," Brazilian Journal of Microbiology, vol. 45, no. 4, pp. 1173-1178, 2014.

50.N. S. Bolan and A. D. Robson, "Increasing phosphorus supply can increase the infection of plant roots by vesicular-arbuscular mycorrhizal fungi," Soil Biology and Biochemistry, vol. 16, pp. 419-420, 1984.

51.F. S. B. Silva, "Fase assimbiótica, produção, infectividade e efetividade de fungos micorrízicos em substratos com adubos orgânicos [PhD Thesis]," Centro de Ciências Biológicas, Recife; pp. 297, 2006.

52.A. Gildon and P. B. Tinker, "A heavy metal-tolerant strain of a mycorrhizal fungus," Transactions of the British Mycological Society, vol. 77, pp. 648-649, 1981.

53.F. M. S. Moreira and J. O. Siqueira, "Micorrizas," in Microbiologia e Bioquímica do Solo. UFLA, Lavras, 2002, pp. $473-539$.

54.M. Luis, I. Carvalho, M. Cacador, and A. Martins-Loucao, "Arbuscular mycorrhizal fungi enhance root cadmium and copper accumulation in the roots of the salt marsh plant Aster tripolium L.," Plant and Soil, vol. 285, no. 1, pp. 161-169, 2006.

55.I. Ortas and C. Akpinar, "Response of kidney bean to arbuscular mycorrhizal inoculation and mycorrhizal dependency in P and Zn deficient soils," Acta Agriculturae Scandinavica, Section B - Plant and Soil Science, vol. 56, pp. 101-109, 2006.

56.S. V. Motha, H. Amballa, and N. R. Bhumi, "Arbuscular mycorrhizal fungi associated with rhizosphere soils of brinjal cultivated in Andhra Pradesh, India," International Journal of Current Microbiologyand Applied Sciences, vol. 3, no. 5, pp. 519-529, 2014.

57.S. E. Smith and D. J. Read, Mycorrhizal symbiosis. Academic Press, San Diego, 1997.

58.H. Vierheilig, B. Bago, C. Albrecht, M. J. Poulin, and Y. Piche, "Flavonoids and arbuscular mycorrhizal fungi," in Flavonoids in the living system. Plenum, New York, 1998a, pp. 9-33.

59.C. Sbrana and M. Giovanetti, "Chemotropism in the arbuscular mycorrhizal fungus Glomus mosseae," Mycorrhiza, vol. 15, no. 7, pp. 539-545, 2005.

60.J. M. Scervino, M. A. Ponce, R. Erra-Bassells, H. Vierheilig, J. A. Ocampo, and A. Godeas, "Flavonoids exhibit fungal species and genus specific effects on the presymbiotic growth of Gigaspora and Glomus," Mycological Research, vol. 109, no. 7, pp. 789-794, 2005b.

61.J. M. Scervino, M. A. Ponce, R. Erra-Bassells, J. Bompadre, H. Vierheilig, J. A. Ocampo, and A. Godeas, "The effect of flavones and flavonols on colonization of tomato plants by arbuscular mycorrhizal fungi of the genera Gigaspora and Glomus," Canadian Journal of Microbiology, vol. 53, pp. 702-709, 2007. 